\title{
Measuring the Directional or Non-Directional Distance between Type-1 and Type-2 Fuzzy Sets with Complex Membership Functions
}

\author{
Josie McCulloch and Christian Wagner
}

\begin{abstract}
Fuzzy sets may have complex, non-normal or nonconvex membership functions that occur, for example, in the output of a fuzzy logic system or when automatically generating fuzzy sets from data. Measuring the distance between such nonstandard fuzzy sets can be challenging as there is no clear correct method of comparison and limited research currently exists that systematically compares existing distance measures for these fuzzy sets. It is useful to know the distance between these sets, which can tell us how much the results of a system change when the inputs differ, or the amount of disagreement between individual's perceptions or opinions on different concepts. In addition, understanding the direction of difference between such fuzzy sets further enables us to rank them, learning if one represents a higher output or higher ratings than another. This paper picks up previous functions of measuring directional distance and, for the first time, presents methods of measuring the directional distance between any type- 1 and type- 2 fuzzy sets with both normal/nonnormal and convex/non-convex membership functions. In realworld applications where data-driven, non-convex, non-normal fuzzy sets are the norm, the proposed approaches for measuring the distance enables us to systematically reason about the realworld objects captured by the fuzzy sets.
\end{abstract}

Index Terms-distance, directional distance, type-2 fuzzy sets, non-normal, non-convex

\section{INTRODUCTION}

As applications centring on employing large-scale data become prolific - in particular on human perceptions, preferences and habits - measures that enable the comparison of comprehensive models (e.g., type-2 fuzzy sets in Computing with Words) are needed. Distance measures are particularly useful as they enable one to understand the relative difference between fuzzy sets (FSs) with respect to their universe of discourse. With improved distance measures, applications such as clustering of type- 2 fuzzy sets can produce more accurate results.

As well as determining the magnitude of distance between the FSs, it is also useful to learn the direction of distance; i.e., establishing if one FS is to the left or right of another. Though some literature on directional distance measures already exists [1], [2], current methods do not have the mathematical properties that would be generally desired.

Additionally, difficulties arise when measuring the distance between non-normal or non-convex FSs. For example, Fig.

LUCID - Lab for Uncertainty in Data and Decision Making, School of Computer Science, University of Nottingham, United Kingdom email: josie.mcculloch; christian.wagner@ nottingham.ac.uk

This work is funded by the EPSRCs DTP - University of Nottingham grant, EP/M50810X/1

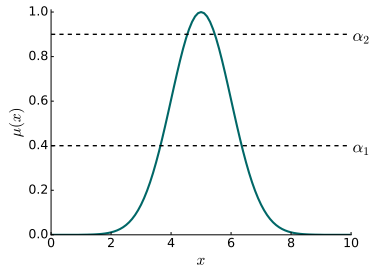

(a)

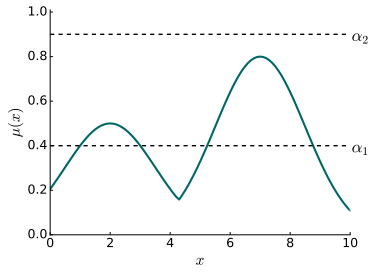

(b)
Fig. 1. A normal unimodal FS (a); and a non-normal bimodal FS (b).

1 shows a unimodal and a bimodal FS representing the aggregated subjective opinions on two variables. The bimodal function is non-convex as people's beliefs have fallen into two distinct groups. It is also non-normal (with a highest degree of membership less than 1) as neither group is certainly correct. To measure the distance between these two sets, a series of horizontal cuts ( $\alpha$-cuts) are commonly compared. However, in some cases (e.g., $\alpha_{1}$ in Fig. 1) the bimodal set results in a noncontinuous interval (effectively, a set of two intervals), and in other cases (e.g., $\alpha_{2}$ ) the cut results in the empty set. This paper examines measuring distance in such complex cases.

Some research has been published into the distance between type-1 (T1) FSs that are non-normal [2], [3] or non-convex [2], but these measures are either non-directional [3] or lack the expected properties of a distance measure [2]. There is also limited literature on the $\alpha$-cut-based distance between interval type-2 (IT2) FSs [4], but this does not account for direction, non-normality or non-convexity in the membership functions. Additionally, to the authors' knowledge, there are currently no $\alpha$-cut-based distance measures to compare general type-2 (T2) FSs. Note that it is important to use $\alpha$-cuts as they enable a comparison of FSs along the $x$-axis.

This paper builds upon existing measures [1], [2], [4] developing a distance measure for T1 FSs that can measure the directional or non-directional distance between FSs that may be non-normal or non-convex; proofs of the resulting measure's properties are presented. The approach is then extended to enable the comparison of T2 FSs. The functions proposed in this paper are available online as part of our toolkit at https://lucidresearch.org/software and at https://bitbucket. org/JosieMcCulloch/fuzzycreator.

Note that in order to determine the distance between FSs, the ordering along the $x$-axis must be taken into account [5]. Therefore, an $\alpha$-cut based approach is the most appropriate. Any measure that accounts for vertical slices only (such as in 
[6]) is more akin to measuring dissimilarity. However, distance by dissimilarity is a fundamentally different measure with different properties that calculates the difference in overlap between FSs. In this paper, we measure the distance between FSs in relation to their universe of discourse; this is measured through $\alpha$-cuts.

Further, note that in principle any measure (including any distance measure) on a set may be designed to itself return an output of the same type of set. For example, a distance measure on T1 FSs would return a T1 FS distance. Such a distance measure is, for example, discussed in [7]. It is clear that returning a set of the same type as the input sets minimises the potential for information loss. However, most commonly, measures on fuzzy sets are designed to return crisp outputs for use in decision making and similar applications. This paper thus focuses specifically on developing (distance) measures returning a crisp output for convex/non-convex, normal/nonnormal T1 and T2 FSs.

It is important to note, however, that a crisp distance value will, of course, capture minimal information about the input FSs. For example, it will not be possible to know from the value of distance if the fuzzy sets compared were non-normal or non-convex. In a future publication, we are looking to revisit some of the contributions made in this paper in respect to more complex (distance) measures which return non-numeric distances, such as proposed in [7].

This paper is structured as follows: Section II presents background on FSs and existing distance measures. Section III presents a directional distance measure for T1 FSs that may be non-convex or non-normal. Then, Sections IV and $\mathrm{V}$ extend the measure to compare interval and general type-2 FSs, respectively. Finally, conclusions are presented in Section VII.

\section{BACKGROUND}

This section presents background on FSs and the key methods for measuring their distance. For reference, Table I provides descriptions of set notations and functions used throughout the paper.

\section{A. Fuzzy Sets}

This section presents the necessary background on FS theory and notations for T1 and T2 FSs.

1) Type-1 Fuzzy Sets:

Definition 1. Let $T 1(X)$ denote the set of all FSs in the universe of discourse $X$. The FS $A \in T 1(X)$ is defined by a set of pairs as

$$
A=\left\{\left(x, \mu_{A}(x)\right) \mid \forall x \in X\right\},
$$

where $\mu_{A}(x) \in[0,1]$ denotes the membership value of $x$ in A.

Definition 2. The height $H_{A}$ of a FS $A$ is its maximum membership value, defined as $\max _{x \in X} \mu_{A}(x)$.

Definition 3. A T1 FS $A \in T 1(X)$ is described as normal if $H_{A}=1.0$; i.e., $\max \left\{\mu_{A}(x) \mid \forall x \in X\right\}=1$. Otherwise, it is non-normal.
TABLE I

TABLE OF NOTATIONS. COMMON FS NOTATIONS ARE OMITTED. NOTE ACRONYMS DM (DISTANCE MEASURE), DDM (DIRECTIONAL DM), UMF (UPPER MEMBERSHIP FUNCTION) AND LMF (LOWER MEMBERSHIP FUNCTION).

\begin{tabular}{lll}
\hline Set notation & Meaning & Ref. \\
\hline$A$ & T1 FS & $(1)$ \\
$\tilde{A}$ & T2 FS & $(6)$ \\
$\tilde{A}_{L}$ & lower membership function of $\tilde{A}$ & $(8)$ \\
$\tilde{A}_{U}$ & upper membership function of $\tilde{A}$ & $(9)$ \\
$\bar{A}$ & continuous interval & $(3)$ \\
$-\bar{H}$ & non-continuous interval & $(5)$ \\
$Z_{\tilde{A}}$ & set of zLevels of $\tilde{A}$ & $(11)$ \\
\hline Function & & \\
\hline$\overline{\boldsymbol{d}}$ & Set of distance functions on intervals & $(12)$ \\
$\bar{d}$ & DM for continuous intervals & $(13)(14)(16)$ \\
-- & DM for non-continuous intervals & $(18)$ \\
$d$ & DM for T1 FSs & $(19)$ \\
$d^{T 1}$ & DM for IT2 FSs & $(21)$ \\
$d^{I T 2}$ & DM for T2 FSs & $(23)$ \\
$d^{T 2}$ &
\end{tabular}

Definition 4. A FS $A$ is convex if and only if [8]

$$
\begin{aligned}
& \forall x_{1} \in X, \forall x_{2} \in X, \forall \lambda \in[0,1], \\
& \mu_{A}\left(\lambda x_{1}+(1-\lambda) x_{2}\right) \geq \min \left\{\mu_{A}\left(x_{1}\right), \mu_{A}\left(x_{2}\right)\right\} .
\end{aligned}
$$

A FS that does not satisfy (2) is non-convex.

Definition 5. An $\alpha$-cut of a convex normal FS $A \in T 1(X)$ is written as [9]

$$
\overline{A_{\alpha}}=\left\{x \mid \mu_{A}(x) \geq \alpha, \alpha \in[0,1]\right\} .
$$

Note that $\bar{A}$ denotes an interval and $A$ denotes a T1 FS. Any $\alpha$-cuts of $A$ above $H_{A}$ will be empty sets.

The $\alpha$-cut of a normal, convex FS can be represented as a continuous interval. Thus, an $\alpha$-cut may be rewritten as

$$
\begin{aligned}
\overline{A_{\alpha}} & =\left[\overline{A_{\alpha}}, \overline{A_{\alpha}}\right] \\
\overline{A_{\alpha}} L & =\min \left\{x \mid \mu_{A}(x) \geq \alpha, \alpha \in[0,1]\right\} \\
\overline{A_{\alpha}} & =\max \left\{x \mid \mu_{A}(x) \geq \alpha, \alpha \in[0,1]\right\}
\end{aligned}
$$

However, this representation changes when FSs are nonconvex or non-normal.

The $\alpha$-cut of a non-convex region within a non-convex FS cannot be described as a continuous interval (as in (4)). Instead, it is described by a set of non-overlapping, continuous intervals. This is defined as a non-continuous interval as follows:

Definition 6. Let a non-continuous interval $\bar{H}$ be [10]

$$
\bar{H}=\bigcup_{i=1}^{I} \bar{H}_{i}
$$

where $\bar{H}_{i}$ represents the $i^{\text {th }}$ continuous interval within $\bar{H}$ and $I$ is the total number of intervals within $\bar{H}$. 
Such non-continuous intervals arise, for example, when describing the $\alpha$-cut of a non-convex FS. For example, referring to Fig. $1, \bar{B}_{\alpha_{1}}^{-}=\{[0.985,3.015],[5.376,8.625]\}$.

2) Type-2 Fuzzy Sets: A T2 FS is an extension of a T1 FS in which the membership value of any element is defined as a T1 FS instead of a crisp number.

Definition 7. Let $T 2(X)$ represent the set of all T2 FSs within $X$, then the FS $\tilde{A} \in T 2(X)$ is formally written in terms of a set of pairs as [11]

$$
\tilde{A}=\left\{\left((x, u), \mu_{\tilde{A}}(x, u)\right) \mid \forall x \in X, u \in[0,1]\right\},
$$

where $x$ is the primary variable in $X, u \in[0,1]$ is the secondary variable, and the amplitude of $\mu_{A}(x, u) \in[0,1]$ is known as the secondary grade.

In addition to (6), many representations of T2 FSs have been developed. This paper uses the zSlices [12] (aka, alphaplane [13]) representation (introduced in Section II-A4). This is based on the theory of IT2 FSs, introduced next.

3) Interval Type-2 Fuzzy Sets: An IT2 FS is a special case of T2 in which each secondary membership value greater than 0 has a membership of 1 .

Definition 8. Let IT $2(X)$ represent the set of all IT2 FSs within $X$. The FS $\tilde{A} \in I T 2(X)$ is formally written as [14]

$$
\begin{aligned}
\tilde{A}=\left\{\left((x, u), \mu_{\tilde{A}}(x, u)=1\right) \mid\right. & \forall x \in X, \\
u & \left.\in\left[\underline{\mu}_{\tilde{A}}(x), \bar{\mu}_{\tilde{A}}(x)\right] \subseteq[0,1]\right\} .
\end{aligned}
$$

where $\underline{\mu}_{\tilde{A}}(x)$ and $\bar{\mu}_{\tilde{A}}(x)$ are defined as the lower and upper membership values of $\tilde{A}$. The lower and upper membership functions of $\tilde{A}$ are

$$
\begin{aligned}
& \tilde{A}_{L}=\left\{(x, u) \mid \min _{u \in[0.1]} \mu_{\tilde{A}}(x, u)>0, \forall x \in X\right\} \\
& \tilde{A}_{U}=\left\{(x, u) \mid \max _{u \in[0.1]} \mu_{\tilde{A}}(x, u)>0, \forall x \in X\right\}
\end{aligned}
$$

Definition 9. The $\alpha$-cut of an IT2 FS may be represented by the $\alpha$-cuts of the upper and lower membership functions; throughout this paper, this is denoted $\tilde{A}_{\alpha}=\left\{\overline{\tilde{A}_{L_{\alpha}}}, \overline{\tilde{A}_{U_{\alpha}}}\right\}$ for $\tilde{A} \in I T 2(X)$ where $\overline{\tilde{A}_{L_{\alpha}}}$ and $\overline{\tilde{A}_{U_{\alpha}}}$ are the $\alpha$-cuts of the lower and upper membership functions of $\tilde{A}$, respectively.

4) zSlices Type-2 Fuzzy Sets : A zSlices T2 FS can be composed by slicing a T2 FS along the $z$-axis, segmenting the FS into many IT2 set-like FSs called $z$ Slices. Each resulting zSlice has a secondary membership of $z_{i}$, referred to as the zLevel.

Definition 10. The zSlice $Z_{i}$ has a secondary membership grade at $z_{i}$ and is defined as [12]

$$
\tilde{Z}_{i}=\left\{\left((x, u), \mu_{\tilde{Z}_{i}}(x, u) \geq z_{i}\right) \mid \forall x \in X, \forall u \in[0,1]\right\} .
$$

A FS $\tilde{F} \in T 2(X)$ can be represented by the union of its zSlices [12].

Definition 11. As a zlices T2 FS $\tilde{A} \in T 2(X)$ is a collection of IT2 FSs, we represent the $\alpha$-cuts of $\tilde{A}$ as the collection of $\alpha$-cuts of its zSlices as given in Definition 9). That is, $\tilde{A}_{z_{i_{\alpha}}}=$
$\left\{\tilde{A}_{z_{i_{L}}}, \tilde{A}_{z_{i_{U}}}\right\}$; i.e. the $\alpha$-cut of $\tilde{A}_{z_{i}}$ is composed of the $\alpha$ cuts of the lower and upper membership functions of $\tilde{A}_{z_{i}}$.

Definition 12. $Z_{\tilde{A}}$ denotes the set of all zLevels of the zSlices in $\tilde{A}$. This is defined as

$$
Z_{\tilde{A}}=\left\{z_{i} \mid \forall i \in\{1,2, \ldots, I\}, \tilde{A}_{z_{i}} \neq\{\}\right\},
$$

where $I$ is the total number of zLevels in $\tilde{A}$.

\section{B. Distance Measures}

A distance measure is a function $d: A \times B \rightarrow \mathbb{R}^{+}$or $\mathbb{R}$ for non-directional and directional distance, respectively, where $A$ and $B$ are crisp sets, or T1 or T2 FSs. This section covers background on non-directional and directional distance measures.

1) Non-directional Distance : Some common properties of a non-directional distance measure include:

Self-Identity: $\quad d(A, B)=0 \Longleftrightarrow A=B$

Symmetry: $d(A, B)=d(B, A)$

Separability: $d(A, B) \geq 0$

Triangle inequality: $d(A, C) \leq d(A, B)+d(B, C)$

Transitivity: If $A \leq B \leq C$, then $d(A, B) \leq d(A, C)$

For transitivity, note that $A \leq B$ if $A_{\alpha} \leq B_{\alpha} \forall \alpha \in[0,1]$.

Note, as mentioned in Section I, we only focus on measures of distance that calculate the difference between fuzzy sets in the $x$-axis by measuring $\alpha$-cuts .

One of the most common methods of calculating the distance between two convex, normal FSs $A, B \in T 1(X)$ by comparing $\alpha$-cuts is the following [1], [3], [4], [15]-[18]:

$$
d^{T 1}(A, B)=\frac{\sum_{\alpha \in(0,1]} \overline{\boldsymbol{d}}\left(A_{\alpha}, B_{\alpha}\right) f(\alpha)}{\sum_{\alpha \in(0,1]} f(\alpha)},
$$

where $\overline{\boldsymbol{d}}$ refers to the set of distance functions used to calculate distance between two intervals ( $\alpha$-cuts). The function $f(\alpha)$ may be used to weight the distance at a given $\alpha$-cut . If so, it is typically a non-negative and increasing function on $[0,1]$ with $f(0)=0, f(1)=1$ and $\int_{0}^{1} f(\alpha) d \alpha=\frac{1}{2}$; in most cases (and in this paper) $f(\alpha)=\alpha$ [3], [18], [19]. However, sometimes a weighting function is not used such that $f(\alpha)=1$ [15]-[17].

Note that $d^{T 1}$ (12) does not measure where $\alpha=0$ as this relates to all elements that are not within the FS.

In this paper, $\bar{d}$ refers to the set of distance functions to compare two intervals, and $\bar{d}$ refers to a specific distance function, as shown next.

Often, the distance between two intervals is some form of the Minkowski [1], [4], [16], [17] or Hausdorff distance [3], [15]. The Minkowski distance $\bar{d}_{r}$ between two intervals $\bar{A}$ and $\bar{B}$ is

$$
\bar{d}_{r}(\bar{A}, \bar{B})=\sqrt[r]{1 / 2\left(\left(\bar{A}_{L}-\bar{B}_{L}\right)^{r}+\left(\bar{A}_{R}-\bar{B}_{R}\right)^{r}\right)} .
$$

where $r>1$ and $\left[\bar{A}_{L}, \bar{A}_{R}\right]$ represents a continuous interval; e.g., the $\alpha$-cut of a FS $A \in T 1(X)$. The Hausdorff distance $\bar{d}_{h}$ between two intervals $\bar{A}$ and $\bar{B}$ is [5]

$$
\bar{d}_{h}(\bar{A}, \bar{B})=\max \left\{\left|\bar{A}_{L}-\bar{B}_{L}\right|,\left|\bar{A}_{R}-\bar{B}_{R}\right|\right\}
$$

To compare non-normal FSs, [3] and [2] present methods based on the Hausdorff distance, where [2] also measures 
distance on non-convex FSs. Extensions using the Minkowski distance are not currently in the literature. These are further developed in Section III.

To compare IT2 FSs, Figueroa-García et al. [4] developed a distance measure using the Minkowski distance (where $r=1$ ) to compare $\alpha$-cuts of the upper and lower membership functions. The distance between two IT2 FSs $\tilde{A}, \tilde{B} \in I T 2(X)$ is given as (12), where $f(\alpha)=\alpha$ and $\bar{d}_{f}$ is used for $\overline{\boldsymbol{d}}$, given as [4]

$$
\begin{aligned}
\bar{d}_{f}\left(\tilde{A}_{\alpha}, \tilde{A}_{\alpha}\right)= & {\left[\left|\overline{\tilde{A}_{\alpha_{i_{U} L}}}-\overline{\tilde{B}_{\alpha_{i_{U} L}}}\right|+\right.} \\
& \left|\overline{\tilde{A}_{\alpha_{i_{W} L}}}-\overline{\tilde{B}_{\alpha_{i_{W} L}}}\right|+ \\
& \left|\overline{\tilde{A}_{\alpha_{i_{W}}}}-\overline{\tilde{B}_{\alpha_{i_{W}}}}\right|+ \\
& \left.\left|\overline{\tilde{A}_{\alpha_{i_{U} R}}}-\overline{\tilde{B}_{\alpha_{i_{U} R}}}\right|\right]
\end{aligned}
$$

where $\overline{\tilde{A}_{\alpha_{i} W}}$ is the $\alpha$-cut of the lower membership function of $\tilde{A}$ (that is, $\overline{\tilde{A}_{\alpha_{i} W}}=\left[\overline{\tilde{A}_{\alpha_{i} W} L}, \overline{\tilde{A}_{\alpha_{i_{W} R}}}\right]$ ), and $\overline{\tilde{A}_{\alpha_{i_{U}}}}$ is the $\alpha$-cut of the upper membership function of $\tilde{A}$.

Note that (15) requires the membership functions of $\tilde{A}$ and $\tilde{B}$ to be convex and normal. However, $\bar{d}_{f}$ could also be used to compare convex, non-normal IT2 FSs with identical heights.

In addition to (15), Figueroa-García et al. [4] also proposed two methods based on comparing the centroids of FSs; for more details on this see [4].

Note that, to the authors' knowledge, there are no current $\alpha$-cut based measures of distance beyond IT2 to general T2 FSs.

2) Directional Distance Measures: A directional distance measure is one that does not follow separability and instead uses a signed result to indicate direction; thus giving a result within $\mathbb{R}$ instead of $\mathbb{R}^{+}$. A directional distance measure has the benefit of indicating if one fuzzy set contains lower or higher values from the universe of discourse than another fuzzy set. This is useful in applications requiring ranking of fuzzy sets, in which ordering is important.

The property of symmetry of a directional distance measure is altered to $d(A, B)=-d(B, A)$ [1]. Some properties of directional distance are presented in [1] and will be further explored in this paper in Section III-A.

Yao and $\mathrm{Wu}[1]$ present a measure where $d(A, B) \geq 0$ if $A \geq B$ and $d(A, B)<0$ if $A<B$, using $d^{T 1}$ (12), where $f(\alpha)=\alpha$ and $\bar{d}$ is

$$
\bar{d}_{y}\left(\overline{A_{\alpha}}, \overline{B_{\alpha}}\right)=\left[\overline{A_{\alpha}}+\overline{A_{\alpha}}-\overline{B_{\alpha}}-\overline{B_{\alpha}}\right] .
$$

In [2], a directional distance measure using the general equation (12) was presented, where $f(\alpha)=\alpha$ and the Hausdorff distance (14) is altered to

$$
\bar{d}_{m}(\bar{A}, \bar{B})= \begin{cases}\bar{B}_{l}-\bar{A}_{l}, & \text { if }\left|\bar{B}_{l}-\bar{A}_{l}\right|>\left|\bar{B}_{r}-\bar{A}_{r}\right| . \\ \bar{B}_{r}-\bar{A}_{r}, & \text { otherwise. }\end{cases}
$$

However, this approach does not account for cases of symmetry where $\left(\bar{A}_{L}-\bar{B}_{L}\right)=-\left(\bar{A}_{R}-\bar{B}_{R}\right)$. For example, if $\bar{A}=[3,5]$ and $\bar{B}=[2,6]$, it is not clear if the distance should be 1 or -1 . Additionally, as a result, (17) does not always follow transitivity or triangle inequality.
This concludes the background of distance measures in the literature. The next section expands upon these current measure to introduce a directional distance measure for T1 FSs that may be non-normal or non-convex.

\section{Directional Distance For Normal, CONVEX, TYPE-1 FUZZY SETS}

This section uses a hybrid of pre-existing measures to propose a new directional distance measure on non-normal and non-convex FSs. The theory of measuring distance for non-normal and non-convex FSs builds on [2]. However, as described with (17), this existing work cannot be applied when comparing $\alpha$-cuts with identical centres but different lengths. This is due to a limitation of using the Hausdorff distance to describe direction. Additionally, the directional Hausdorff distance does not have the property of triangle inequality, which may make it unsuitable for many applications. As an alternative, the Manhattan-based directional distance proposed by Yao and $\mathrm{Wu}$ [1] is used. Using a hybrid of these methods ensures the resulting directional distance measure has predictable properties that are consistent with non-directional measures.

This section first discusses the properties of (16) [1], after which the measure is extended for non-convex or non-normal FSs.

\section{A. Properties of Directional Distance}

Due to the directional nature of $\bar{d}_{y}$ (16), the standard properties of a distance measure are slightly altered. Some of these properties are discussed within [1] and this section explores additional properties in more detail.

1) Self-Identity: If two FSs are identical then their distance is zero, as is standard with the non-directional form of the Manhattan distance. However, this is not the only case in which the distance may be zero.

For two intervals, $\bar{A}$ and $\bar{B}$, if one interval is a subset of the other and the distances $\left(\bar{A}_{L}-\bar{B}_{L}\right)$ and $-\left(\bar{A}_{R}-\bar{B}_{R}\right)$ are equal then their directional distance is zero. We shall denote this property reflectivity.

Definition 13 (Reflectivity). The distance between two intervals is 0 if the distances between their respective end points are equal to each other and in opposite directions.

$\bar{d}_{y}(\bar{A}, \bar{B})=0$ if $\left(\bar{A}_{L}-\bar{B}_{L}\right)=-\left(\bar{A}_{R}-\bar{B}_{R}\right)$, where $\bar{A}=\left[\bar{A}_{L}, \bar{A}_{R}\right]$ and $\bar{B}=\left[\bar{B}_{L}, \bar{B}_{R}\right]$

For example, in Fig. 2, $\bar{A}=[1,7]$ and $\bar{B}=[3,5]$. Given that $\bar{A}_{L}-\bar{B}_{L}=-2$ and $\bar{A}_{R}-\bar{B}_{R}=2$, the resulting distance $\bar{d}(\bar{A}, \bar{B})$ is 0 .

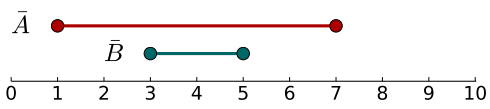

Fig. 2. Two intervals, $\bar{A}$ and $\bar{B}$, where $\bar{B}$ is a subset of $\bar{A}$ and $\bar{d}(\bar{A}, \bar{B})=0$.

Theorem 1. $\bar{d}_{y}(A, B)=0$ if $A=B$.

Proof: If $\bar{A}=\bar{B}$ then $\bar{A}_{L}-\bar{B}_{L}=\bar{A}_{R}-\bar{B}_{R}=0$ thus $\bar{d}_{y}(\bar{A}, \bar{B})=0$ 
Theorem 2. $\bar{d}_{y}$ (16) follows reflectivity.

Proof:

$$
\begin{gathered}
\text { Let } \beta=\bar{A}_{L}-\bar{B}_{L}=-\left(\bar{A}_{R}-\bar{B}_{R}\right) \\
\begin{aligned}
\bar{d}_{y}(\bar{A}, \bar{B}) & =\bar{A}_{L}-\bar{B}_{L}+\bar{A}_{R}-\bar{B}_{R} \\
& =\beta+(-\beta) \\
& =0
\end{aligned}
\end{gathered}
$$

2) Symmetry: The directional distance produces signed results such that $d(A, B)=-d(B, A)$, thus the property of symmetry does not hold. Instead, we introduce a new property denoted partial-symmetry.

Definition 14 (Partial Symmetry). Let partial-symmetry describe the property of a distance measure $d: A \times B \rightarrow \mathbb{R}$ for two points or objects $A$ and $B$ as

$$
d(A, B)=-d(B, A) .
$$

This property enables us to indicate both the magnitude of the distance (by the absolute value) and the direction of distance (by the sign). Note, this property also appears in [1].

Theorem 3. $\bar{d}_{y}$ (16) follows partial-symmetry.

Proof:

$$
\begin{aligned}
\bar{d}_{y}(\bar{A}, \bar{B}) & =-\bar{d}_{y}(\bar{B}, \bar{A}) \\
\bar{A}_{L}-\bar{B}_{L}+\bar{A}_{R}-\bar{B}_{R} & =-\left(\bar{B}_{L}-\bar{A}_{L}+\bar{B}_{R}-\bar{A}_{R}\right) \\
& =-\bar{B}_{L}+\bar{A}_{L}-\bar{B}_{R}+\bar{A}_{R}
\end{aligned}
$$

3) Separability: The property of separability no longer holds as any real negative or non-negative value may result from the measure. Instead, we define a new form of separability denoted directional-separability.

Definition 15 (Directional-separability). The sign of the distance indicates the relative positions between the variables.

$$
\begin{array}{ll}
d(A, B)<0 & \text { if } A<B \\
d(A, B) \geq 0 & \text { if } A \geq B,
\end{array}
$$

For directional separability, we must define when $\bar{A}<\bar{B}$. Several methods of interval order have been proposed in literature [20], of which we choose some common methods: $\bar{A}<\bar{B} \Leftrightarrow$ :

- $\bar{A}_{R}<\bar{B}_{L}$

- $\bar{A}_{L}<\bar{B}_{L}$ and $\bar{A}_{R}<\bar{B}_{R}$

- $\bar{A}_{M}<\bar{B}_{M}$ and $\bar{A}_{W}<\bar{B}_{W}$

- $\bar{A}_{M}<\bar{B}_{M}$ and $\bar{A}_{L}<\bar{B}_{L}$

- $\bar{A}_{M}<\bar{B}_{M}$ and $\bar{A}_{R}<\bar{B}_{R}$

where $\bar{A}_{M}=\left(\bar{A}_{L}+\bar{A}_{R}\right) / 2$ and $\bar{A}_{W}=\bar{A}_{R}-\bar{A}_{L}$. We reduce the last three to $\bar{A}_{M}<\bar{B}_{M} \Rightarrow \bar{A}<\bar{B}$ and show that this is sufficient to prove directional-separability for these three definitions of interval ordering.

Theorem 4. $\bar{d}_{y}$ (16) follows directional-separability.

Proof:

When $\bar{A}<\bar{B} \Leftrightarrow \bar{A}_{R}<\bar{B}_{L}$

Then $\bar{A}_{L}-\bar{B}_{L}<0$ and $\bar{A}_{R}-\bar{B}_{R}<0$
Therefore $\bar{d}_{y}(\bar{A}, \bar{B})<0$ if $\bar{A}<\bar{B}$.

When $\bar{A}<\bar{B} \Leftrightarrow \bar{A}_{L}<\bar{B}_{L}$ and $\bar{A}_{R}<\bar{B}_{R}$

If $\bar{A}_{L}<\bar{B}_{L}$ then $A_{L}-B_{L}<0$

and if $\bar{A}_{R}<\bar{B}_{R}$ then $A_{R}-B_{R}<0$

then $\bar{A}_{L}+\bar{A}_{R}-\bar{B}_{L}-\bar{B}_{R}<0$, thus $\bar{d}_{y}(\bar{A}, \bar{B})<0$

When $\bar{A}_{M}<\bar{B}_{M} \Rightarrow \bar{A}<\bar{B}$

It is given that $\bar{A}_{M}=\frac{\bar{A}_{L}+\bar{A}_{R}}{2}$

Therefore $\frac{\bar{A}_{L}+\bar{A}_{R}}{2}<\frac{\bar{B}_{L}+\bar{B}_{R}}{2} \Rightarrow \bar{A}<\bar{B}$

and $\bar{A}_{L}+\bar{A}_{R}^{2}<\bar{B}_{L}+\stackrel{2}{B_{R}} \Rightarrow \bar{A}<\bar{B}$

$\bar{A}_{L}+\bar{A}_{R}-\left(\bar{B}_{L}+\bar{B}_{R}\right)<0$ thus $\bar{d}_{y}(\bar{A}, \bar{B})<0$

In each case, the likewise is given for $\bar{d}_{y}(\bar{A}, \bar{B}) \geq 0$ if $\bar{A} \geq \bar{B}$

4) Triangle Inequality: In a non-directional distance measure, because $\bar{d}(\bar{A}, \bar{B})=\bar{d}(\bar{B}, \bar{A})$, the ordering of the given intervals that are measured has no effect on the rule of triangle inequality, e.g., both

$$
\bar{d}(\bar{A}, \bar{C}) \leq \bar{d}(\bar{A}, \bar{B})+\bar{d}(\bar{B}, \bar{C})
$$

and

$$
\bar{d}(\bar{A}, \bar{C}) \leq \bar{d}(\bar{B}, \bar{A})+\bar{d}(\bar{B}, \bar{C})
$$

are true. However, for the directional distance, it is necessary to consider the ordering of the FSs when applying the rule of triangle inequality. This can be explained with the aid of Table II. If the first input of part 1 appears as the first input where it occurs in part 2, then the distance is unchanged. Otherwise, the negative of the result must be used. Likewise, if the second input of part 1 appears as the second input where it occurs in part 2 the sign is kept the same. Otherwise, the negative of the result is used.

TABLE II

AN EXAMPLE OF THE RESTRICTED PROPERTY OF TRIANGLE INEQUALITY ON THE DIRECTIONAL DISTANCE MEASURE $\bar{d}_{y}(16)$.

\begin{tabular}{crr}
\hline Part 1 & & \multicolumn{1}{c}{ Part 2 } \\
\hline $\bar{d}_{y}(\bar{A}, \bar{C})$ & $\leq$ & $\bar{d}_{y}(\bar{A}, \bar{B})+\bar{d}_{y}(\bar{B}, \bar{C})$ \\
$\bar{d}_{y}(\bar{A}, \bar{C})$ & $\leq$ & $-\bar{d}_{y}(\bar{B}, \bar{A})+\bar{d}_{y}(\bar{B}, \bar{C})$ \\
$\bar{d}_{y}(\bar{A}, \bar{C})$ & $\leq$ & $\bar{d}_{y}(\bar{A}, \bar{B})-\bar{d}_{y}(\bar{C}, \bar{B})$ \\
$\bar{d}_{y}(\bar{A}, \bar{C})$ & $\leq$ & $-\bar{d}_{y}(\bar{B}, \bar{A})-\bar{d}_{y}(\bar{C}, \bar{B})$ \\
\hline
\end{tabular}

Note that the restricted triangle inequality is not affected by the ordering of the FSs, i.e., $\bar{d}_{y}(\bar{A}, \bar{C}) \leq \bar{d}_{y}(\bar{A}, \bar{B})+\bar{d}_{y}(\bar{B}, \bar{C})$ is true if $\bar{A} \leq \bar{B} \leq \bar{C}$, or $\bar{B} \leq \bar{A} \leq \bar{C}$, or any other ordering on $\bar{A}, \bar{B}$ and $\bar{C}$.

5) Transitivity: The property of transitivity works in the opposite direction to usual; i.e., if $A \leq B \leq C$, instead of $\bar{d}(\bar{A}, \bar{B}) \leq \bar{d}(\bar{A}, \bar{C})$ it follows that $\overline{d_{y}}(\bar{A}, \overline{\bar{B}}) \geq \bar{d}_{y}(\bar{A}, \bar{C})$. However, the magnitude of distance (ignoring sign) follows the normal rule; i.e. $\left|\bar{d}_{y}(\bar{A}, \bar{B})\right| \leq\left|\bar{d}_{y}(\bar{A}, \bar{C})\right|$.

Theorem 5. For $\bar{A} \leq \bar{B} \leq \bar{C}, \bar{d}_{y}(\bar{A}, \bar{B}) \geq \bar{d}_{y}(\bar{A}, \bar{C})$. 
Proof:

$$
\begin{aligned}
\bar{d}_{y}(\bar{A}, \bar{B}) & \geq \bar{d}_{y}(\bar{A}, \bar{C}) \\
\bar{A}_{L}-\bar{B}_{L}+\bar{A}_{R}-\bar{B}_{R} & \geq \bar{A}_{L}-\bar{C}_{L}+\bar{A}_{R}-\bar{C}_{R} \\
-\bar{B}_{L}-\bar{B}_{R} & \geq-\bar{C}_{L}-\bar{C}_{R} \\
\bar{B}_{L}+\bar{B}_{R} & \leq \bar{C}_{L}+\bar{C}_{R}
\end{aligned}
$$

Given that $\bar{B} \leq \bar{C}$ it follows that $\bar{d}_{y}(\bar{A}, \bar{B}) \geq \bar{d}_{y}(\bar{A}, \bar{C})$.

The remainder of this section presents methods of measuring the distance between T1 FSs that may have non-convex or non-normal membership functions. The methods proposed enable one to measure either the directional or non-directional distance between such sets.

\section{B. Non-Convex Fuzzy Sets}

To measure the distance (directional or non-directional) between non-convex FSs, a method of comparing non-continuous intervals is required. For example, referring to Fig. 1, at $\alpha_{1}$, the FS in Fig. 1(a) results in a continuous interval. However, in 1(b) it is represented by a non-continuous interval because the FS is non-convex.

Definition 16 (Distance between non-continuous intervals). As described in [2], the directional distance between $\alpha$-cuts that may be non-continuous is calculated as

$$
\bar{d}\left(\overline{A_{\alpha}}, \overline{B_{\alpha}}\right)=\frac{1}{n m} \sum_{i=1}^{n} \sum_{j=1}^{m} \overline{\boldsymbol{d}}\left(\overline{A_{\alpha_{i}}}, \overline{B_{\alpha_{j}}}\right)
$$

where $\overline{A_{\alpha_{i}}}$ represents the $i^{\text {th }}$ continuous interval within $\overline{A_{\alpha}}$, and $n$ and $m$ are the total number of continuous intervals within $\overline{A_{\alpha}}$ and $\overline{B_{\alpha}}$, respectively. The function $\overline{\boldsymbol{d}}$ denotes the set of directional distance measures on intervals.

In (18), we take the average distance between discontinuous intervals, but a weighted average can implemented if, for example, close regions of FSs are more important than far regions.

The function $\bar{d}$ may be (16) for directional distance, and $\bar{d}_{r}^{n c}$ (13) and $\bar{d}_{h}^{n c}$ (14) for non-directional distance. In each case, $\bar{d}$ inherits the properties of the chosen method for $\overline{\boldsymbol{d}}$.

The function $\bar{d}\left(\overline{A_{\alpha}}, \overline{B_{\alpha}}\right)$ compares the distance (using a function from $\overline{\boldsymbol{d}}$ ) of every continuous interval within $\bar{A}_{\alpha}$ against every continuous interval in $\overline{B_{\alpha}}$. If both $\overline{A_{\alpha}}$ and $\overline{B_{\alpha}}$ each contain one continuous interval (i.e., they are convex) then only one comparison is made.

Note that $\bar{d}$ can compare non-continuous intervals (from non-convex FSs) but cannot be used to compare empty intervals (from non-normal FSs).

Consider three $\alpha$-cuts, where $A_{\alpha}=[2,4], B_{\alpha}=$ $([3,5],[7,9])$ and $C_{\alpha}=[5,7]\left(A_{\alpha}\right.$ and $C_{\alpha}$ and convex, $B_{\alpha}$ is non-convex). Using (18), $\bar{d}\left(A_{\alpha}, B_{\alpha}\right)=\bar{d}\left(A_{\alpha}, C_{\alpha}\right)$; the distance is the same even though the $\alpha$-cuts are fundamentally different. While this may not be desired, it will always be an issue when simplifying the distance of fuzzy sets to a

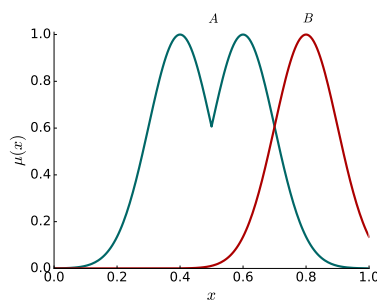

(a) (b)

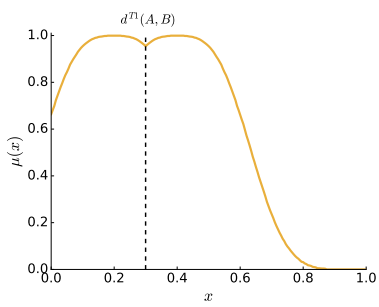

Fig. 3. A non-convex fuzzy set and a convex fuzzy set (a); and the distance between these fuzzy sets as a fuzzy set (orange) and as a singleton using (18) (black dashed).

singleton. If it necessary to show non-convexity in the distance when it is present in the fuzzy sets that are compared, we recommend modelling distance as a fuzzy set [7].

When distance must be represented as a singleton, we recommend the proposed method (18) for measuring non-convex fuzzy sets. Although the most accurate result is achieved by representing distance as a fuzzy set, a singleton result is usually required in most applications. The defuzzified result of the distance represented by a fuzzy set provides an appropriate singleton approximation. Our proposed measure provides the centroid of the fuzzy distance. For example, Fig. 3 shows two fuzzy sets and their distance as a fuzzy set (using [7]) and as a singleton (using (18)). Note that the singleton result is the centroid of the fuzzy result. This example shows that the proposed method provides a good singleton approximation of the distance between non-convex fuzzy sets. (Note that the methods in [7] only provide fuzzy-valued distance for T1 FSs, not T2).

\section{Non-Normal FSs}

For two fuzzy sets $A$ and $B$, if $H_{A}=H_{B}$ (such as for normal fuzzy sets) only parallel $\alpha$-cuts should be compared, i.e., we should not measure $\bar{d}\left(A_{\alpha_{i}}, B_{\alpha_{j}}\right)$ where $i \neq j$. If we do, then $d(A, B) \neq 0$ when $A=B$ because $\alpha$-cuts of a fuzzy set are usually different at different $\alpha$ levels. Next, consider $A$ and $B$ where $H_{A}<H_{B}<1$. There exist $\alpha$-cuts where $A_{\alpha}=\emptyset$ and $B_{\alpha} \neq \emptyset$ (where $H_{A}<\alpha \leq H_{B}$ ) and $\alpha$-cuts where $A_{\alpha}=B_{\alpha}=\emptyset$ (where $\alpha>H_{B}$ ). However, if we only measure parallel $\alpha$-cuts then we ignore $B$ where $H_{A}<\alpha \leq$ $H_{B}$.

We propose measuring $\bar{d}\left(A_{H_{A}}, B_{\alpha}\right)$ where $H_{A}<\alpha \leq H_{B}$ so that a full comparison of the fuzzy sets can be made (comparing $\alpha$-cuts in $B$ with the highest possible cut in $A$ ). Instead of weighting this distance with $f(\alpha)=\alpha$ as is done in (12), we weight the distance at these $\alpha$-cuts as $H_{A}$. This is chosen because $A$ does not have values with membership greater than $H_{A}$ and therefore the distance $\bar{d}\left(A_{H_{A}}, B_{\alpha}\right)$ cannot be any more confident than $H_{A}$. We also propose that the distance where $\alpha>\max \left\{H_{A}, H_{B}\right\}$ is not measured since both fuzzy sets are empty at these $\alpha$-cuts.

Considering the above, the distance between $A$ and $B$ is measured as

$$
d^{T 1}(A, B)=\frac{\sum_{\alpha \in(0, \lambda]} \overline{\boldsymbol{d}}\left(A_{\min \left\{H_{A}, \alpha\right\}}, B_{\min \left\{H_{A}, \alpha\right\}}\right) f(\alpha)}{\sum_{\alpha \in(0, \lambda]} f(\alpha)},
$$


where $\lambda=\max \left\{H_{A}, H_{B}\right\}$ and $f(\alpha)=\min \left\{H_{A}, H_{B}, \alpha\right\}$.

To compare FSs that are both non-normal and non-convex, $d^{T 1}(A, B)(19)$ can be used with $\bar{d}$ (18). We propose (19) (for normal and non-convex FSs) as an alternative to (12) (for normal, convex FSs).

Theorem 6. $d^{T 1}$ has the properties self-identity, symmetry, separability, triangle inequality and transitivity when used with a non-directional distance measure for two intervals.

Proof: For self-identity, symmetry and separability, the proofs are trivial.

Triangle Inequality and Transitivity:

As the measure is a weighted average for each used value of $\alpha$, increasing or decreasing the number of $\alpha$-cuts compared does not increase or decrease the calculated distance according to $d^{T 1}$. Thus, the properties are retained.

Theorem 7. $d^{T 1}$ has the properties self-identity, partialsymmetry and directional-separability when used with a directional distance measure for two intervals.

Proof: The proofs are trivial.

This concludes the introduction of a new directional distance measure on T1 FSs. This improves upon the existing directional distance measures [1], [2] by enabling the comparison of non-normal and non-convex FSs whilst maintaining the expected properties of a directional distance measure as described in Section III-A. The next section extends this measure to IT2 FSs.

\section{Distance on Interval Type-2 Fuzzy Sets}

A T1 FS can be modelled as an IT2 FS where $\mu(x)=$ $\bar{\mu}(x) \forall x \in X$. Based on this, we would expect the distance between T1 FSs using an IT2 representation and IT2 distance measure to be equal to their distance using the T1 representation and measure. This ensures that the results of T1 and T2 measures can be easily compared because the type of FS does not affect the interpretation of the results.

We measure the distance between IT2 FSs $\tilde{A}$ and $\tilde{B}$ by comparing their UMFs and their LMFs, and aggregating the two results. We do this based on the method of comparing T1 FSs. As the upper and lower MFs of IT2 FSs are T1 FSs, let $A$ be a T1 embedded set of $\tilde{A}$ and let the distance between two embedded MFs (UMFs or LMFs) of $\tilde{A}, \tilde{B} \in I T 2(X)$ be

$$
d_{L U}^{I T 2}(A, B)=\sum_{\alpha \in[0, \gamma]} f(\alpha) \overline{\boldsymbol{d}}\left(\bar{A}_{\min \left\{H_{A}, \alpha\right\}}, \bar{B}_{\min \left\{H_{B}, \alpha\right\}}\right)
$$

where $\gamma=\max \left\{H_{A}, H_{B}\right\}$ and $f(\alpha)=\min \left\{H_{A}, H_{B}, \alpha\right\}$. Note that at this stage (20) the average distance across $\alpha$ cuts is not normalised the same as for T1 FSs (19). Next, we aggregate the distance between upper and lower MFs and normalise the distance together.

Definition 17 (Interval Type-2 Distance Measure). The directional distance between two FSs $\tilde{A}, \tilde{B} \in I T 2(X)$ may be measured by comparing the upper and lower membership functions as

$$
d^{I T 2}(\tilde{A}, \tilde{B})=\frac{d_{L U}^{I T 2}\left(A_{U}, B_{U}\right)+d_{L U}^{I T 2}\left(A_{L}, B_{L}\right)}{\sum_{\alpha \in\left[0, \gamma_{U}\right]} f_{U}(\alpha)+\sum_{\alpha \in\left[0, \gamma_{L}\right]} f_{L}(\alpha)}
$$

where $f_{U}(\alpha)=\min \left\{H_{A_{U}}, H_{B_{U}}, \alpha\right\}$ (where $H_{A_{U}}$ is the height of the upper membership function of $\tilde{A})$ and $f_{L}(\alpha)=$ $\min \left\{H_{A_{L}}, H_{B_{L}}, \alpha\right\}$.

The function $d^{I T 2}$ (21) enables the comparison of nonnormal IT2 FSs. In addition, depending on the choice of interval distance for $\overline{\boldsymbol{d}}$ within $d_{L U}^{I T 2}(20)$, it is possible to compare non-convex FSs and the result may be either directional or non-directional.

Theorem 8. $d^{I T 2}$ has the properties self-identity, symmetry, separability, triangle inequality and transitivity when used with a non-directional distance measure for two intervals.

Proof: The proofs are trivial.

Theorem 9. $d^{I T 2}$ has the properties self-identity, partialsymmetry and directional-separability when used with a directional distance measure for two intervals.

Proof: The proofs are trivial.

\section{Distance on Type-2 Fuzzy Sets}

A distance measure on T2 FSs is attained by using the IT2 distance measure $d^{I T 2}$ on the zSlices of the FSs at relative zlevels and aggregating the results. This is a method that has previously been applied to extend IT2 similarity measures to T2 FSs [21]-[24].

We propose measuring the distance between zSlices based fuzzy sets by only comparing zSlices at equal zLevels where possible. However, it is possible for T2 FSs to have nonnormal membership functions within the secondary membership axis. This may occur, for example, when there is no consensus when modelling agreement between individuals. Figure 4 shows an example of a FS that has non-normal secondary membership functions $(\tilde{A})$ and a FS that is normal ( $\tilde{B})$.

To compare such sets as those in figure 4, we take the same approach as for non-normal T1 FSs. The distance between zSlices is measured at equal zLevels where both FSs are nonempty at the given zLevel. At zLevels where one FS $\tilde{A}_{z}$ is empty and another $\tilde{B}_{z}$ is not, the highest non-empty zLevel of $\tilde{A}$ is measured against $\tilde{B}_{z}$. At zLevels where both FSs are empty, distance is not measured.

We use $L(\tilde{A}, \tilde{B})$ to denote the maximum zLevel that can be used to compare $\tilde{A}$ and $\tilde{B}$.

\section{Definition 18.}

$$
\begin{aligned}
L(\tilde{A}, \tilde{B})=\{z \mid & z \in Z_{\tilde{A}} \cup Z_{\tilde{B}}, \\
z & \left.\leq \min \left(\max \left(Z_{\tilde{A}}\right), \max \left(Z_{\tilde{B}}\right)\right)\right\}
\end{aligned}
$$

where $Z_{\tilde{A}}$ is described in (11).

Definition 19. The distance between two T2 FSs $\tilde{A}, \tilde{B} \in$ $G T 2(X)$ may be measured as

$$
d^{T 2}(\tilde{A}, \tilde{B})=\frac{\sum_{i \in L(A, B)} z_{i} d^{I T 2}\left(\tilde{A}_{z_{i}}, \tilde{B}_{z_{i}}\right)}{\sum_{i \in L(A, B)} z_{i}},
$$

where $d^{I T 2}$ is the distance measure on IT2 FSs (21) and $L(\tilde{A}, \tilde{B})$ is give in (22). 


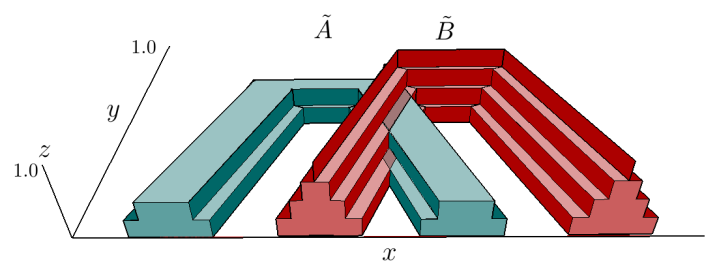

Fig. 4. Two FSs $\tilde{A}, \tilde{B} \in G T 2(X)$ where $\tilde{A}$ is non normal $\left(Z_{\tilde{A}}=\right.$ $\{0.25,0.5\})$, and $\tilde{B}\left(Z_{\tilde{B}}=\{0.25,0.5,0.75,1.0\}\right)$.

Using $L(\tilde{A}, \tilde{B})$ ensures that all zSlices up to the lowest maximum zLevel, even if the FSs are divided into different zLevels [22]. Note that $d^{T 2}$ inherits the properties of $d^{I T 2}$, including the ability to measure either directional or nondirectional distance.

Theorem 10. $d^{G T 2}$ has the properties self-identity, symmetry, separability, triangle inequality and transitivity when used with a non-directional distance measure for two intervals.

Proof: For self-identity, symmetry and separability, the proofs are trivial.

Triangle Inequality and Transitivity:

As the measure is a weighted average for each used value of $z$, increasing or decreasing the number of zLevels compared does not increase or decrease the calculated distance according to $d^{G T 2}$. Thus, the properties are retained.

Theorem 11. $d^{G T 2}$ has the properties self-identity, partialsymmetry and directional-separability when used with a directional distance measure for two intervals.

Proof: The proofs are trivial.

This concludes the proposed methods of measuring directional and non-directional distance on T1 and T2 FSs that may have non-normal or non-convex primary membership functions and, for T2 FSs, non-normal secondary membership functions. Note that although non-convex secondary membership functions are possible, they are uncommon due to their complexity, and are not considered further in this paper. Further, we note that (23) enables the direct comparison on T2 (interval and general), as well as T1 FSs.

\section{NUMERICAL EXAMPLES}

This section demonstrates the proposed directional distance measures on T1, IT2 and GT2 FSs. Each example includes three fuzzy sets:

- $A$ : non-normal and convex

- $B$ : non-normal and non-convex

- $C$ : normal and convex

We calculate the distance using four $\alpha$-cuts at $\alpha \in\{0.25,0.5,0.75,1.0\}$.

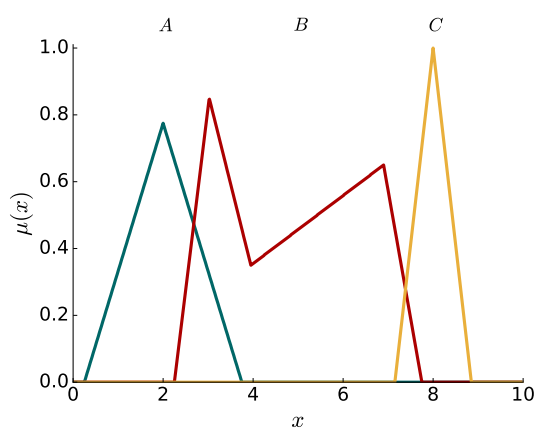

Fig. 5. Type-1 fuzzy sets.

\section{A. Type-1 Fuzzy Sets}

The FSs are defined by key values below and linear interpolation is used to calculate intermediary membership values.

$$
\begin{aligned}
& A=\{(0.25,0),(2,0.775),(3.75,0)\} \\
& B=\{(2.25,0),(3.025,0.85),(3.95,0.35), \\
&(6.9,0.65),(7.75,0)\} \\
& C=\{(7.15,0),(8,1),(8.85,0)\}
\end{aligned}
$$

Fig. 5 illustrates the FSs. The $\alpha$-cuts are given in Table III. The distance between the FSs at each $\alpha$-cut and overall are given in Table IV.

TABLE III

$\alpha$-CUTS OF FSS IN (24) AND FIG. 5.

\begin{tabular}{llll}
\hline$\alpha$ & $A$ & $B$ & $C$ \\
\hline 1 & - & - & $(8.000,8.000)$ \\
0.75 & {$[1.944,2.056]$} & {$[2.940,3.210]$} & {$[7.788,8.212]$} \\
0.5 & {$[1.379,2.621]$} & $([2.710,3.670]$, & {$[7.575,8.425]$} \\
& & $[5.430,7.090])$ & \\
0.25 & {$[0.815,3.185]$} & {$[2.480,7.420]$} & {$[7.362,8.638]$} \\
\hline
\end{tabular}

TABLE IV

DisTANCE BETWEEN T1 FSS IN (24) AND FIG. 5 AT INDIVIDUAL $\alpha$-CUTS AND OVERALL.

\begin{tabular}{lllll}
\hline$\alpha$ & $d(A, C)$ & $d(C, A)$ & $d(B, C)$ & $d(A, B)$ \\
\hline 1.0 & -6.0 & 6.0 & -4.925 & - \\
0.75 & -6.0 & 6.0 & -4.925 & -1.075 \\
0.5 & -6.0 & 6.0 & -3.275 & -2.725 \\
0.25 & -6.0 & 6.0 & -3.050 & -2.950 \\
Overall & -6.0 & 6.0 & -4.062 & -1.938 \\
\hline
\end{tabular}

As $A$ and $C$ are symmetrical $\mathrm{FSs}$, their distance is equivalent to comparing their centres of gravity. Note the symmetry in distance, such that $d(A, C)=-d(C, A)$.

Comparing $B$ with $C$, their distance is larger at higher $\alpha$ cuts, particularly at $\alpha=0.75$ where the right-most region of the non-convex FS $B$ is not present. Lower $\alpha$-cuts of $B$ are closer to $C$, to the extent of overlapping; this is reflected in the calculated distance that is smaller at lower $\alpha$-cuts. The distance between $A$ and $B$ decreases at lower $\alpha$-cuts at $B$ begins to overlap $A$. 


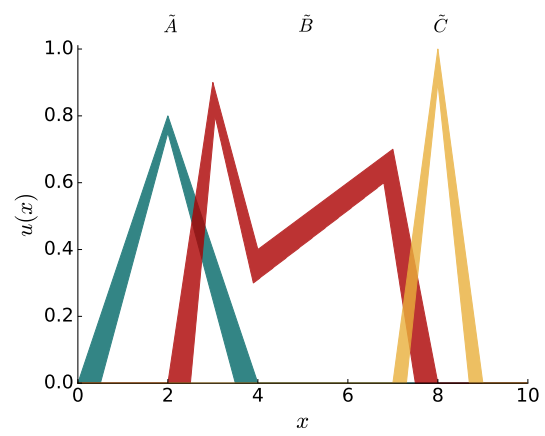

Fig. 6. Type-2 fuzzy sets.

\section{B. Interval Type-2 Fuzzy Sets}

$$
\begin{aligned}
& A_{U}=\{(0,0),(2,0.8),(4,0)\} \\
& A_{L}=\{(0.5,0),(2,0.75),(3.5,0)\} \\
& B_{U}=\{(2,0),(3,0.9),(4,0.4),(7,0.7),(8,0)\} \\
& B_{L}=\{(2.5,0),(3.05,0.9),(3.9,0.4),(6.8,0.7),(7.5,0)\} \\
& C_{U}=\{(7,0),(8,1),(9,0)\} \\
& C_{L}=\{(7.3,0),(8,1),(8.7,0)\}
\end{aligned}
$$

Fig. 6 illustrates the fuzzy sets. The $\alpha$-cuts are given in Table V.

TABLE V

$\alpha$-CUTS OF FSS IN (25) AND FIG. 6.

\begin{tabular}{llll}
\hline$\alpha$ & $A$ & $B$ & $C$ \\
\hline 1 & - & - & {$[8.000,8.000]$} \\
0.75 & {$[1.875,2.125]$} & {$[2.840,3.300]$} & {$[7.750,8.250]$} \\
0.5 & {$[1.250,2.750]$} & $([2.560,3.800]$, & {$[7.500,8.500]$} \\
& & $[5.000,7.280])$ & \\
0.25 & {$[0.625,3.375]$} & {$[2.280,7.640]$} & {$[7.250,8.750]$} \\
\hline
\end{tabular}

TABLE VI

DISTANCE BETWEEN IT2 FSS IN (25) AND FIG. 6 AT INDIVIDUAL $\alpha$-CUTS (FOR LOWER AND UPPER MEMBERSHIP FUNCTIONS, RESPECTIVELY) AND OVERALL.

\begin{tabular}{lllll}
\hline & $d(\tilde{A}, \tilde{C})$ & $d(\tilde{C}, \tilde{A})$ & $d(\tilde{B}, \tilde{C})$ & $d(\tilde{A}, \tilde{B})$ \\
\hline 1.0 & $-6.0,-6.0$ & $6.0,6.0$ & $-4.930,-4.925$ & - \\
0.75 & $-6.0,-6.0$ & $6.0,6.0$ & $-4.930,-4.925$ & $-1.070,-1.075$ \\
0.5 & $-6.0,-6.0$ & $6.0,6.0$ & $-3.340,-3.213$ & $-2.660,-2.788$ \\
0.25 & $-6.0,-6.0$ & $6.0,6.0$ & $-3.040,-3.060$ & $-2.960,-2.940$ \\
Overall & -6.0 & 6.0 & -4.064 & -1.936 \\
\hline
\end{tabular}

The same trend in results can be found in the IT2 results as in the T1 results (Table IV). Specifically, $d(A, C)$ is consistent throughout all $\alpha$-cuts, $d(B, C)$ decreases at lower $\alpha$-cuts, and $d(A, B)$ increases at lower $\alpha$-cuts . The reasons are the same as discussed for Table IV.

\section{General Type-2 Fuzzy Sets}

In this example, we construct a general type-2 fuzzy set with two zSlices. The lowest zSlice $z_{1}$, with a secondary membership (zLevel) of 0.5 is defined by the interval type- 2 fuzzy sets as given in (25) and has the same corresponding $\alpha$ cuts given in Table $\mathrm{V}$. The highest zSlice $z_{2}$, with a secondary

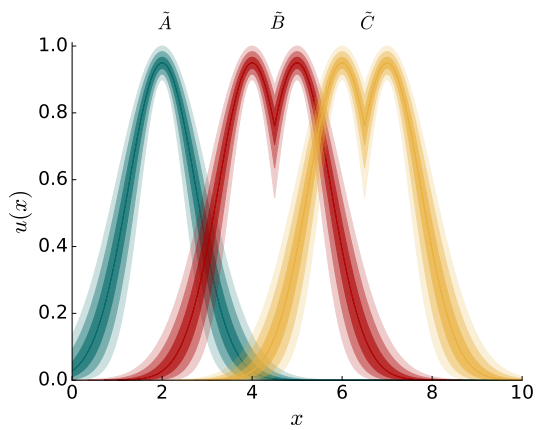

Fig. 7. A convex GT2 FS $(A)$ and two identically shaped non-convex GT2 FSs $(B$ and $C)$ at different locations.

membership of 1.0 is defined by the type-1 fuzzy set given in (24) and $\alpha$-cuts given in Table III.

TABLE VII

DistANCE BETWEen GT2 FSS IN Fig. 6 BASED ON (25) AND (24).

\begin{tabular}{lllll}
\hline & $d(\tilde{A}, \tilde{C})$ & $d(\tilde{C}, \tilde{A})$ & $d(\tilde{B}, \tilde{C})$ & $d(\tilde{A}, \tilde{B})$ \\
\hline$z_{1}$ & -6.0 & 6.0 & -4.064 & -1.936 \\
$z_{2}$ & -6.0 & 6.0 & -4.062 & -1.938 \\
Overall & -6.0 & 6.0 & -4.063 & -1.937 \\
\hline
\end{tabular}

The distance between the FSs is calculated through the weighted average of the distances at $z_{1}$ and at $z_{2}$ with weights 0.5 and 1.0, respectively. The resulting distance at each zSlice and overall distance are given in Table VII. Note that the distances at $z_{1}$ and $z_{2}$ are the same as those for the IT2 and T1 FSs in Tables VI and IV, respectively. The trend in results for GT2 FSs in Table VII are the same as those discussed for the $\mathrm{T} 1$ results (Table IV).

Next, we demonstrate comparing GT2 FSs with non-normal secondary membership functions. As in the previous example, the lowest zSlice $z_{1}$ (at zLevel 0.5 ) is defined by the IT2 FSs given in (25). However, in this example, set $\tilde{A}$ is only described by this single zSlice. Therefore the set of zLevels of $\tilde{A}$ is $Z_{\tilde{A}}=\{0.5\}$. As in the previous example, $\tilde{B}$ and $\tilde{C}$ have a second zSlice $z_{2}$ (at zLevel 1.0) with the same membership functions as the T1 FSs $B$ and $C$ described in (24).

Table VIII shows results of comparing these FSs using $d^{G T 2}$ (23). Note that $L(\tilde{A}, \tilde{B})=\{0.5\}, L(\tilde{A}, \tilde{C})=\{0.5\}$, and $L(\tilde{B}, \tilde{C})=\{0.5,1.0\}$. Therefore, in each case except $d^{G T 2}(\tilde{B}, \tilde{C})$, the distance at only one zLevel can be compared. The results in Table VIII are intuitive with those in Table VII where all FSs contain two zSlices.

TABLE VIII

DISTANCE BETWEEN GT2 FSS IN FIG. 6 WITH NON-NORMAL SECONDARY MEMBERSHIP FUNCTIONS BASED ON (25) AND (24).

\begin{tabular}{lllll}
\hline & $d(\tilde{A}, \tilde{C})$ & $d(\tilde{C}, \tilde{A})$ & $d(\tilde{B}, \tilde{C})$ & $d(\tilde{A}, \tilde{B})$ \\
\hline$z_{1}$ & -6.0 & 6.0 & -4.064 & -1.936 \\
$z_{2}$ & - & - & -4.062 & - \\
Overall & -6.0 & 6.0 & -4.063 & -1.936 \\
\hline
\end{tabular}

Next, we present an example of the behaviour of measuring distance with non-convex T2 FSs. Fig. 7 shows a convex T2 FS $(\tilde{A})$ and two identically shaped non-convex T2 FSs $(\tilde{B}$ and $\tilde{C})$. The modes of $\tilde{B}$ are at $x=4$ and $x=5$, and the FS is 
centred at $x=4.5$. The FS $\tilde{C}$ is the same but shifted by 2 along the $x$-axis; that is, its centre is at $x=6.5$. Using our proposed measure, $d^{T 2}(\tilde{A}, \tilde{B})=2.5$ and $d^{T 2}(\tilde{A}, \tilde{C})=4.5$. These results demonstrate that the proposed measure correctly shows the difference in position of $\tilde{B}$ and $\tilde{C}$ with respect to $\tilde{A}$ and correctly identifies the centres of the FSs. While this result contains limited information in the sense that, being numeric, it does not show the non-convexity in the result (as would be possible when returning a FS valued distance, for instance), it provides a useful single-valued measure of distance. As discussed with Fig. 3, a FS result could convey the non-convexity of the T2 FSs, but loss of such information is unavoidable when reducing a result from its FS form to a numeric (singleton) form.

\section{CONCLUSIONS}

Distance measures are vital in applications centring on employing human perceptions, preferences and habits. They provide the ability to understand the relative difference between FSs with respect to their universe of discourse. Directional distance measures are especially useful in understanding the direction of difference between such FS, for example in ranking, to determine if one FS represents a higher output or higher ratings than another.

Current methods in the literature enable the directional or non-directional distance between T1 FSs. However, although some steps have been made towards comparing non-normal and non-convex FSs, the measures do not have all of the properties of a distance measure, making them difficult to apply. In addition, current distance measures on T2 FSs only exist for IT2 FSs that are normal and convex. Thus, current methods in the literature are not useful in cases where nonnormal and non-convex FSs are common, such as applications with data-driven FSs, for which any measures used on these FSs need be able to handle such circumstances.

Given this, we present extensions to distance measures with the goal of enabling the comparison of different types of realworld FSs. We introduce a new measure on the directional and non-directional distance for $\mathrm{T} 1$ and $\mathrm{T} 2 \mathrm{FSs}$ that may be normal/non-normal and convex/non-convex. Note that for T2 FSs, the extensions in this paper support non-normal secondary MFs, but do not support non-convex secondary MFs. (This will be addressed in a future publication.)

In addition, the measures resulting from the proposed approach are 'backwards-compatible' in the sense that they allow for the direct comparison of GT2 to IT2 and T1 FSs - regardless of the sets' (non-)convexity, (non-)normality and type. As non-standard (e.g. non-convex) fuzzy sets are common in realworld applications where FSs are data-driven, the proposed extensions thus significantly expand the applicability of FSs (including T2 FSs) to applications beyond fuzzy logic, e.g., distance based recommendation systems, as used commonly in marketing.

The functions proposed in this paper are available online as part of our toolkit, available at https://lucidresearch. org/software and at https://bitbucket.org/JosieMcCulloch/ fuzzycreator

\section{REFERENCES}

[1] J.-S. Yao and K. Wu, "Ranking fuzzy numbers based on decomposition principle and signed distance," Fuzzy Sets and Systems, vol. 116, no. 2, pp. 275-288, 2000.

[2] J. McCulloch, C. Wagner, and U. Aickelin, "Measuring the directional distance between fuzzy sets," in Computational Intelligence (UKCI), 2013 13th UK Workshop on, 2013, pp. 38-45.

[3] B. Chaudhuri and A. Rosenfeld, "On a metric distance between fuzzy sets," Pattern Recognition Letters, vol. 17, no. 11, pp. 1157-1160, 1996.

[4] J. C. Figueroa-García, Y. Chalco-Cano, and H. Román-Florez, "Distance measures for Interval Type-2 fuzzy numbers," Discrete Applied Mathematics, vol. 197, pp. 93-102, 2015.

[5] R. Zwick, E. Carlstein, and D. V. Budescu, "Measures of similarity among fuzzy concepts: A comparative analysis," International Journal of Approximate Reasoning, vol. 1, no. 2, pp. 221-242, 1987.

[6] H. Bustince, E. Barrenechea, and M. Pagola, "Relationship between restricted dissimilarity functions, restricted equivalence functions and normal EN-functions: Image thresholding invariant," Pattern Recognition Letters, vol. 29, no. 4, pp. 525-536, 2008. [Online]. Available: http://www.sciencedirect.com/science/article/pii/S0167865507003686

[7] J. McCulloch, C. Hinde, C. Wagner, and U. Aickelin, "A fuzzy directional distance measure," in Fuzzy Systems (FUZZ-IEEE), 2014 IEEE International Conference on, Jul. 2014, pp. 141-148.

[8] L. Zadeh, "Fuzzy sets," Information and Control, vol. 8, no. 3, pp. 338 353, 1965.

[9] —-, "The concept of a linguistic variable and its application to approximate reasoning-I,' Information Sciences, vol. 8, no. 3, pp. 199249, 1975.

[10] C. Wagner, D. T. Anderson, and T. C. Havens, "Generalization of the fuzzy integral for discontinuous interval-and non-convex interval fuzzy set-valued inputs," in Fuzzy Systems (FUZZ), 2013 IEEE International Conference on. IEEE, 2013, pp. 1-8.

[11] J. Mendel and R. John, "Type-2 fuzzy sets made simple," Fuzzy Systems, IEEE Transactions on, vol. 10, no. 2, pp. 117-127, Apr. 2002.

[12] C. Wagner and H. Hagras, "Toward General Type-2 Fuzzy Logic Systems Based on zSlices," Fuzzy Systems, IEEE Transactions on, vol. 18, no. 4, pp. 637-660, Aug. 2010.

[13] J. Mendel and F. Liu, "On new quasi-type-2 fuzzy logic systems," in Fuzzy Systems, 2008. FUZZ-IEEE 2008. (IEEE World Congress on Computational Intelligence). IEEE International Conference on, Jun. 2008, pp. 354-360.

[14] J. Mendel, R. John, and F. Liu, "Interval Type-2 Fuzzy Logic Systems Made Simple,' Fuzzy Systems, IEEE Transactions on, vol. 14, no. 6, pp. 808-821, Dec. 2006.

[15] A. L. Ralescu and D. A. Ralescu, "Probability and fuzziness," Information Sciences, vol. 34, no. 2, pp. 85-92, 1984.

[16] A. Ban, "Approximation of fuzzy numbers by trapezoidal fuzzy numbers preserving the expected interval," Fuzzy Sets and Systems, vol. 159, no. 11 , pp. 1327-1344, 2008

[17] P. Grzegorzewski, "Metrics and orders in space of fuzzy numbers," Fuzzy Sets and Systems, vol. 97, no. 1, pp. 83-94, 1998.

[18] L. Tran and L. Duckstein, "Comparison of fuzzy numbers using a fuzzy distance measure," Fuzzy sets and Systems, vol. 130, no. 3, pp. 331-341, 2002.

[19] T. Allahviranloo, S. Abbasbandy, and R. Saneifard, "A method for ranking of fuzzy numbers using new weighted distance," Mathematical and Computational Applications, vol. 16, no. 2, p. 359, 2011.

[20] A. Sengupta and T. K. Pal, "On comparing interval numbers," European Journal of Operational Research, vol. 127, no. 1, pp. 28-43, 2000.

[21] H. Hamrawi and S. Coupland, "Measures of uncertainty for type-2 fuzzy sets," in Computational Intelligence (UKCI), 2010 UK Workshop on, Sept 2010, pp. 1-6.

[22] J. McCulloch, C. Wagner, and U. Aickelin, "Extending similarity measures of interval type-2 fuzzy sets to general type-2 fuzzy sets," in Fuzzy Systems (FUZZ), 2013 IEEE International Conference on, 2013, pp. 1-8.

[23] T. Zhao, J. Xiao, Y. Li, and X. Deng, "A new approach to similarity and inclusion measures between general type-2 fuzzy sets," Soft Computing, vol. 18, no. 4, pp. 809-823, 2014.

[24] M. Hao and J. M. Mendel, "Similarity measures for general type-2 fuzzy sets based on the $\alpha$-plane representation," Information Sciences, vol. 277, no. 0, pp. 197-215, 2014. 